\title{
Examination of Metformin Effect on Breast Cancer Cell Migration
}

\author{
Farzaneh Afzali ${ }^{1}$, Mossa Gardaneh ${ }^{1, *}$ \\ ${ }^{1}$ Department of Stem Cells and Regenerative Medicine, National Institute of Genetic \\ Engineering and Biotechnology, Tehran, Iran \\ ${ }^{*}$ Corresponding Author: Mossa Gardaneh, Department of Stem Cells and \\ Regenerative Medicine, National Institute of Genetic Engineering and \\ Biotechnology, Tehran, Iran, E-mail:Mossabenis65@gmail.com
}

DOI: $10.21859 /$ mci-supp-65

\section{Keywords:}

MCF7

Breast Cancer

Metformin

Migration

\begin{abstract}
Introduction: Tumor invasion and metastasis are the main driving forces behind cancer-related mortality. Various proteolytic compounds take part in corruption of environmental barriers, such as the ECM and basement membrane. Matrix metalloproteinases (MMPs) as an endopeptidase assumes an essential role in the proteolysis of different ECM components and participates in metastasis development. The oral anti-diabetic medication Metformin (Met) is a bi-guanide compound and globally the most broadly utilized anti-hyperglycemic drug. Reports indicate that Met can potentially induce cancer cell migration, invasion and growth through various cascades and molecules. It has been revealed that Met has an impact on MMPs down-regulation.
\end{abstract}

Materials and Methods: Breast cancer cell line MCF7 was treated with serial concentrations of Met to first examine its efficacy on cell growth by MTT viability assay. Then its effect on inhibiting migration was assessed by the wound healing assay.

Results: Our preliminary data demonstrates that cell death is induced by Met. Besides, we have observed that Met inhibits migration within a $24 \mathrm{~h}$ assessment timeline.

Conclusions: It is revealed that Metformin can be considered as breast cancer cell growth inhibitor, cell death inducer and migration inhibitor. 